\title{
Invasive Alien Plants of Indian Himalayan Region- Diversity and Implication
}

\author{
K. Chandra Sekar
}

G. B. Pant Institute of Himalayan Environment \& Development, Almora, India.

Email: kcsekar1312@rediffmail.com

Received June $10^{\text {th }}, 2011$; revised October $24^{\text {th }}, 2011$; accepted November $15^{\text {th }}, 2011$

\begin{abstract}
The present study deals with comprehensive list of Invasive alien plants of Indian Himalayan Region with background information on family, habit and nativity. A total of 190 invasive alien species under 112 genera, belonging to 47 families have been recorded. Among these, the dicotyledons represent by 40 families, 95 genera and 170 species; monocotyledons represent by 7 families, 17 genera and 20 species. The analysis of invasive species reveals that 18 species have been introduced intentionally, while the remaining species established unintentionally through trade. In terms of nativity, amongst 13 geographic regions, the majority of invasive plants reported from American continent (73\%). While in life form analysis, the herbs (148 species) are dominant, followed by shrubs (19 species), Grass (11 species), Trees (4 species), sedges and climber (3 species each). Most of the invasive species are annual habit (63\%). Apart from these, 90 species (47\%) are being used by locals for medicinal purposes. A better planning is needed for early detection to control and reporting of infestations of spread of new and naturalized weeds to be monitored.
\end{abstract}

Keywords: Invasive Alien Species; Indian Himalayan Region; Nativity; India

\section{Introduction}

The Himalaya, is an enchantment territory of the nature where the magnificence of the world's highest mountains is mirrored in the rugged beauty and unique culture of the people who live in their shadow. The Indian part of Himalayas covering an area about 5 lakh $\mathrm{km}^{2}$ (about $16.2 \%$ of country's total geographical area) and forms the northern boundary of the country. It extends between latitudes $26^{\circ} 20^{\prime}$ and $35^{\circ} 40^{\prime}$ North, and between longitudes $74^{\circ} 50^{\prime}$ and $95^{\circ} 40^{\prime}$ East. The Indian Himalayan Region (IHR) is spreading on 10 states (administrative regions) namely, Jammu \& Kashmir, Himachal Pradesh, Uttaranchal, Sikkim, Arunachal Pradesh, Meghalaya, Nagaland, Manipur, Mizoram, Tripura, and hill regions of 2 states viz., Assam and West Bengal of Indian Republic. Starting from foot-hills in the south (Siwaliks) the region extends to Tibetan plateau in the north (trans-Himalaya) comprising about 95 districts of the country and contributes about $16.2 \%$ of India's total geographical area [1]. The most of IHR area is covered by snow-clad peaks, glaciers of higher Himalaya, dense forest cover of midHimalaya. A total of 10,000 plant species are estimated in IHR, of which 3160 species are endemic [2]. There is no record about the invasive species found in IHR.

Alien species are exotic organisms that occur outside their natural adapted ranges and dispersal potential [3].
Many alien species supports our farming and forestry systems in bulk. However, a few alien species become invasive when they are introduced intentionally or unintentionally outside from their natural habitats into new areas where they express the capability to establish, invade and out-compete native species [4,5]. International Union for Conservation of Nature and Natural Resources (IUCN) defines alien invasive species as an alien species which becomes established in natural or semi-natural ecosystems or habitat, an agent of change, and threatens to native biological diversity. These invasive are widely distributed among all categories of living organisms as well as all kinds of ecosystems throughout the world.

Invasive species cause loss of biodiversity including species extinctions, changes in hydrology and ecosystem function [6]. The exotic plant species vary from native one, based on their requirements, modes of resource acquisition and more consumption which may cause changes in soil structure, its profile, decomposition, nutrient content, moisture, etc. It results significant undesirable impacts on the biodiversity and ecosystems. Thus, Invasive species are a serious hindrance for conservation and sustainable use of biodiversity. Biological invasions now operated on global level and it will undergo rapid increase due to increasing globalization of markets, rise in global trade, travel and tourism [7]. Therefore, it is very 
important to make an effective implication management of invasive species, knowledge about their diversity, life form, habitat, uses, is essential. Considering these reasons, the present study has been selected to enumerate the alien species of Indian Himalayan Region and its diversity with future implication of the region.

\section{Materials and Methods}

Intensive field studies were conducted to record the maximum number of Invasive species in different habitats, i.e. agricultural lands, wastelands, protected areas, river banks, reserve forests, etc. in different states of Uttarakhand. The information available in the literature [821] were collected and in the locality of species in IHR were confirmed through consulting the herbaria of Botanical Survey of India, Dehradun (BSD), Forest Research Institute, Dehradun (DD) and Central National Herbarium, Kolkata (CAL).

The nativity of the invasive plants was recorded from the published literature [22-35]. The modes of introduction of these species in Indian Himalayan Region (IHR) were recorded from the published literature and categorized as food, fodder, medicinal, ornamental, etc. Plants were categorized by life form (herb, undershrub, shrub, climber and tree) and habit (annual and perennial). Habitat (wasteland, cultivated field, river banks, forest, roadside, aquatic, etc.) where a given species was most abundant also noted and parasitic plants were also recorded. Literature and herbarium were consulted to find out use value, if any, of these species. The invasive species are enumerated alphabetically in tabular form, followed by author's abbreviations, name of the Family, Nativity, Life form, Habit, Uses, Habitat and mode of Introduction.

\section{Results}

A total of 190 species distributed in 112 genera and 47 families recorded as invasive alien in the flora of Indian Himalayan Region (Table 1). Among these, the dicotyledons represent by 40 families, 95 genera and 170 species; monocotyledons represent by 7 families, 17 genera and 20 species. Only 18 species namely, Ageratum conyzoides, Cassia alata, Catharanthus pusillus, Celosia argentea, Chenopodium album, Eichhornia crassipes, Impatiens balsamina, Ipomoea eriocarpa, I. quamoclit, Lantana camera, Leucaena latisiliqua, L. leucocephala, Melilotus alba, Mirabilis jalapa, Passiflora foetida, Pennisetum purpureum, Portulaca oleracea and Prosopis juliflora are seem to have been introduced deliberately; the rest of them unintentionally through trade exchange including grain import.

A total of 13 different geographic regions in terms of nativity are recorded in the present study. Among these, about $90 \%$ are contributed by four major geographical regions, viz., continent of America (140 sp.), Africa (20 sp.), Europe (7 sp.) and Mediterranean (4 sp.). The American continents contributed majority (73\%) of noxious invasive plants in IHR. The Africa and Europe contribute 10 and 3 percent respectively. Annuals comprise about 63\% (120 sp.) and the remaining are perennials. Herbs constitute $77 \%$ (148 plants), whereas trees were represented by only four species viz., Acacia dealbata, A. farnesiana, Borassus flabellifer and Leucaena latisiliqua; three climbers namely, Antigonon leptopus and Merremia aegyptia, Solanum seaforthianum have been recorded apart from 19 Shrubs (Table 1 and Figure 1). Three sedges recorded as an invasive species namely, Cyperus difformis, C. iria and Fuirena ciliaris. Apart from these, 11 species of grasses are also recorded as an Invasive of IHR (Table 1). While analyzing the plant habit, $63 \%$ are annuals and $37 \%$ are perennials. The genera with the highest number of alien invasive species in IHR are Ipomoea (10 species); Cassia (9 sp.); Alternanthera, Corchorus and Solanum (5 sp. each); Cleome,

Table 1. Invasive species of Indian Himalayan Region.

\begin{tabular}{|c|c|c|c|c|c|c|c|c|}
\hline SN & Name of the Species & Family & Nativity & $\begin{array}{l}\text { Life } \\
\text { Form }\end{array}$ & Habit & Uses & Habitat & $\begin{array}{l}\text { Mode of } \\
\text { Introduction }\end{array}$ \\
\hline 1 & Acacia dealbata Link & Mimosaceae & Australia & $\mathrm{T}$ & $\mathrm{P}$ & Nk & AR & $\mathrm{Ui}$ \\
\hline 3 & Acacia mearnsii De Wild. & Mimosaceae & Australia & $\mathrm{T}$ & $\mathrm{P}$ & So & $\mathrm{AR}$ & $\mathrm{O}$ \\
\hline 4 & Acanthospermum hispidum DC. & Asteraceae & Brazil & $\mathrm{H}$ & A & M & $\mathrm{W}$ & $\mathrm{Ui}$ \\
\hline 6 & Aeschnomene americana L. & Fabaceae & Trop. America & $\mathrm{H}$ & A & M & AR & $\mathrm{Ui}$ \\
\hline 7 & Ageratum conyzoides L. & Asteraceae & Trop. America & $\mathrm{H}$ & A & M & $\mathrm{W}$ & $\mathrm{O}$ \\
\hline 8 & Ageratum houstonianum Mill. & Asteraceae & Trop. America & $\mathrm{H}$ & A & $\mathrm{Ch}$ & W & $\mathrm{Ui}$ \\
\hline 9 & Alternanthera paronychioides St. Hill. & Amaranthaceae & Trop. America & $\mathrm{H}$ & $\mathrm{P}$ & M & $\mathrm{RB}$ & $\mathrm{Ui}$ \\
\hline 10 & Alternanthera philoxeroides (Mart.) Griseb. & Amaranthaceae & Trop. America & $\mathrm{H}$ & $\mathrm{P}$ & $\mathrm{Nk}$ & $\mathrm{RB}$ & $\mathrm{Ui}$ \\
\hline 11 & Alternanthera pungens Humb., Bonpl. \& Kunth & Amaranthaceae & Trop. America & $\mathrm{H}$ & $\mathrm{P}$ & $\mathrm{M}, \mathrm{Ch}$ & $\mathrm{W}$ & $\mathrm{Ui}$ \\
\hline
\end{tabular}




\section{Continued}

\begin{tabular}{|c|c|c|c|c|c|c|c|c|}
\hline$\overline{13}$ & Alternanthera tenella Colla & Amaranthaceae & Trop. America & $\mathrm{H}$ & $\mathrm{A}$ & $\mathrm{M}$ & $\mathrm{W}$ & $\mathrm{Ui}$ \\
\hline 14 & Amaranthus spinosus L. & Amaranthaceae & Trop. America & $\mathrm{H}$ & A & $\mathrm{M}, \mathrm{V}, \mathrm{Fo}$ & $\mathrm{CF}$ & $\mathrm{Ui}$ \\
\hline 15 & Anagallis arvensis L. & Primulaceae & Europe & $\mathrm{H}$ & A & M & $\mathrm{CF}$ & $\mathrm{Ui}$ \\
\hline 16 & Antigonon leptopus Hook. \& Arn. & Polygonaceae & Trop. America & $\mathrm{C}$ & $\mathrm{P}$ & $\mathrm{M}$ & AR & $\mathrm{Ui}$ \\
\hline 17 & Argemone mexicana L. & Papaveraceae & S. America & $\mathrm{H}$ & A & $\mathrm{M}$ & W & $\mathrm{Ui}$ \\
\hline 18 & Argemone ochroleuca Sweet & Papaveraceae & S. America & $\mathrm{H}$ & A & M & AR, W & $\mathrm{Ui}$ \\
\hline 19 & Asclepias curassavica L. & Asclepiadaceae & Trop. America & $\mathrm{H}$ & $\mathrm{P}$ & M & AR & $\mathrm{Ui}$ \\
\hline 20 & Asphodelus tenuifolius Cav. & Liliaceae & Trop. America & $\mathrm{H}$ & A & M & $\mathrm{CF}$ & $\mathrm{Ui}$ \\
\hline 21 & Bidens pilosa L. & Asteraceae & Trop. America & $\mathrm{H}$ & A & M, V, Fo & $\mathrm{CF}$ & $\mathrm{Ui}$ \\
\hline 22 & Blainvillea acmella (L.f.) Philipson & Asteraceae & Trop. America & $\mathrm{H}$ & A & $\mathrm{Ch}$ & $\mathrm{W}$ & $\mathrm{Ui}$ \\
\hline 23 & Blumea eriantha DC. & Asteraceae & Trop. America & $\mathrm{H}$ & $\mathrm{P}$ & $\mathrm{Ch}$ & W & $\mathrm{Ui}$ \\
\hline 24 & Blumea lacera (Burm. F.) DC. & Asteraceae & Trop. America & $\mathrm{H}$ & A & M & W & $\mathrm{Ui}$ \\
\hline 25 & Blumea obliqua (L.) Druce & Asteraceae & Trop. America & $\mathrm{H}$ & A & $\mathrm{Ch}$ & W & $\mathrm{Ui}$ \\
\hline 26 & Borassus flabellifer L. & Arecaceae & Trop. Africa & $\mathrm{T}$ & $\mathrm{P}$ & $\mathrm{Hu}, \mathrm{Hf}$ & $\mathrm{W}$ & $\mathrm{Ui}$ \\
\hline 27 & Bromus catharticus Vahl & Poaceae & S. America & G & A & Fo & $\mathrm{CF}, \mathrm{W}$ & $\mathrm{Ui}$ \\
\hline 29 & Calotropis procera (Ait.) R. Br. & Asclepiadaceae & Trop. Africa & $\mathrm{S}$ & $\mathrm{P}$ & $\mathrm{Ne}, \mathrm{St}, \mathrm{M}$ & $\mathrm{W}$ & $\mathrm{Ui}$ \\
\hline 30 & Cannabis sativa $\mathrm{L}$. & Cannabaceae & Central Asia & $\mathrm{H}$ & $\mathrm{P}$ & $\begin{array}{l}\text { R, C, Sc, } \\
\text { M, Cn, Fi }\end{array}$ & AR, W & $\mathrm{Ui}$ \\
\hline 31 & Cardamine hirsuta L. & Brassicaceae & Trop. America & $\mathrm{H}$ & $\mathrm{P}$ & $\mathrm{Nk}$ & $\mathrm{RB}$ & $\mathrm{Ui}$ \\
\hline 32 & Cardamine trichocarpa Hochst. ex A. Rich. & Brassicaceae & Trop. America & $\mathrm{H}$ & A & Fo, V & $\mathrm{W}$ & $\mathrm{Ui}$ \\
\hline 33 & Cassia absus L. & Caesalpiniaceae & Trop. America & $\mathrm{H}$ & A & M & $\mathrm{W}$ & $\mathrm{Ui}$ \\
\hline 34 & Cassia alata L. & Caesalpiniaceae & S. America & $\mathrm{S}$ & $\mathrm{P}$ & M & $\mathrm{CF}$ & $\mathrm{O}$ \\
\hline 35 & Cassia hirsuta L. & Caesalpiniaceae & Trop. America & $\mathrm{H}$ & A & Fo & $\mathrm{W}$ & $\mathrm{Ui}$ \\
\hline 36 & Cassia obtusifolia L. & Caesalpiniaceae & Trop. America & $\mathrm{H}$ & $\mathrm{P}$ & $\mathrm{M}$ & $\mathrm{W}$ & $\mathrm{Ui}$ \\
\hline 37 & Cassia occidentalis L. & Caesalpiniaceae & S. America & $\mathrm{H}$ & $\mathrm{P}$ & $\mathrm{M}$ & $\mathrm{W}$ & $\mathrm{Ui}$ \\
\hline 38 & Cassia pumila Lam. & Caesalpiniaceae & Trop. America & $\mathrm{H}$ & $\mathrm{A}$ & $\mathrm{Ch}$ & $\mathrm{W}$ & $\mathrm{Ui}$ \\
\hline 39 & Cassia rotundifolia Pers. & Caesalpiniaceae & S. America & $\mathrm{H}$ & A & Fo & $\mathrm{W}$ & $\mathrm{Ui}$ \\
\hline 40 & Cassia tora $\mathrm{L}$. & Caesalpiniaceae & S. America & $\mathrm{H}$ & A & M & $\mathrm{W}$ & $\mathrm{Ui}$ \\
\hline 41 & Cassia uniflora Mill. & Caesalpiniaceae & S. America & $\mathrm{H}$ & A & Fo & $\mathrm{W}$ & $\mathrm{Ui}$ \\
\hline 42 & Catharanthus pusillus (Murr.) G. Don & Apocynaceae & Trop. America & $\mathrm{H}$ & A & Po & $\mathrm{CF}$ & $\mathrm{O}$ \\
\hline 43 & Celosia argentea $\mathrm{L}$. & Amaranthaceae & Trop. Africa & $\mathrm{H}$ & A & $\mathrm{M}, \mathrm{V}$ & $\mathrm{CF}$ & Fd \\
\hline 46 & Chenopodium ambrosioides L. & Chenopodiaceae & Trop. America & $\mathrm{H}$ & A & $\mathrm{Nk}$ & $\mathrm{W}$ & $\mathrm{Ui}$ \\
\hline 47 & Chenopodium murale L. & Chenopodiaceae & Trop. America & $\mathrm{H}$ & A & $\mathrm{V}$ & $\mathrm{CF}, \mathrm{W}$ & $\mathrm{Ui}$ \\
\hline 48 & Chloris barbata (L.) Sw. & Poaceae & Trop. America & G & $\mathrm{P}$ & Fo & $\mathrm{W}$ & $\mathrm{Ui}$ \\
\hline 49 & Chrozophora plicata (Vahl) A. Juss. ex Spreng. & Euphorbiaceae & Trop. Africa & $\mathrm{H}$ & A & $\mathrm{Nk}$ & $\mathrm{W}$ & $\mathrm{Ui}$ \\
\hline 50 & Cleome *iscose L. & Capparaceae & Trop. America & $\mathrm{H}$ & A & M, Cn & $\mathrm{W}$ & $\mathrm{Ui}$ \\
\hline 51 & Cleome gynandra L. & Capparaceae & Trop. America & $\mathrm{H}$ & A & $\mathrm{M}, \mathrm{V}$ & $\mathrm{W}$ & $\mathrm{Ui}$ \\
\hline 52 & Cleome monophylla L. & Capparaceae & Trop. Africa & $\mathrm{H}$ & A & We & AR & $\mathrm{Ui}$ \\
\hline 53 & Cleome rutidosperma DC. & Capparaceae & Trop. America & $\mathrm{H}$ & A & $\mathrm{M}$ & $\mathrm{AR}$ & $\mathrm{Ui}$ \\
\hline 54 & Clidemia hirta (L.) D. Don & Melastomataceae & Trop. America & $\mathrm{H}$ & A & $\mathrm{Nk}$ & AR & $\mathrm{Ui}$ \\
\hline 55 & Convolvulus arvensis L. & Convolvulaceae & Europe & $\mathrm{H}$ & A & M & F, W & $\mathrm{Ui}$ \\
\hline 56 & Conyza *anadensis (L.) Cronquist & Asteraceae & S. America & $\mathrm{H}$ & A & $\mathrm{Ch}$ & $\mathrm{F}$ & $\mathrm{Ui}$ \\
\hline 57 & Corchorus aestuans L. & Tiliaceae & Trop. America & $\mathrm{H}$ & A & M & $\mathrm{W}$ & $\mathrm{Ui}$ \\
\hline 58 & Corchorus fascicularis Lam. & Tiliaceae & Trop. America & $\mathrm{H}$ & A & $\mathrm{M}$ & $\mathrm{W}$ & $\mathrm{Ui}$ \\
\hline 59 & Corchorus olitorius L. & Tiliaceae & Trop. Africa & $\mathrm{H}$ & A & M & $\mathrm{CF}$ & $\mathrm{Ui}$ \\
\hline 60 & Corchorus tridens L. & Tiliaceae & Trop. Africa & $\mathrm{H}$ & A & $\mathrm{V}, \mathrm{Fi}, \mathrm{Ne}$ & AR, W & $\mathrm{Ui}$ \\
\hline 61 & Corchorus trilocularis L. & Tiliaceae & Trop. Africa & $\mathrm{H}$ & A & $\mathrm{Ch}$ & $\mathrm{W}$ & $\mathrm{Ui}$ \\
\hline 62 & Crassocephalum crepidioides (Benth.) S. Moore & Asteraceae & Trop. America & $\mathrm{H}$ & A & $\mathrm{Ch}$ & $\mathrm{F}$ & $\mathrm{Ui}$ \\
\hline 63 & Crotalaria pallida Ait. & Fabaceae & Trop. America & $\mathrm{H}$ & A & $\mathrm{Ch}$ & $\mathrm{CF}$ & $\mathrm{Ui}$ \\
\hline 64 & Crotalaria retusa $\mathrm{L}$. & Fabaceae & Trop. America & $\mathrm{H}$ & A & $\mathrm{Ch}$ & $\mathrm{CF}$ & $\mathrm{Ui}$ \\
\hline 65 & Croton bonplandianum Baill. & Euphorbiaceae & S. America & $\mathrm{H}$ & $\mathrm{P}$ & $\mathrm{Ch}$ & $\mathrm{W}$ & $\mathrm{Ui}$ \\
\hline 66 & Cryptostegia grandiflora R. Br. & Asclepiadaceae & Madagascar & $\mathrm{H}$ & $\mathrm{P}$ & $\mathrm{O}$ & $\mathrm{CF}$ & $\mathrm{Ui}$ \\
\hline 67 & Cuscuta chinensis Lam. & Cusutaceae & Mediterranean & $\mathrm{H}$ & $\mathrm{P}$ & M & $\mathrm{P}$ & $\mathrm{Ui}$ \\
\hline 68 & Cuscuta reflexa Roxb. & Cusutaceae & Mediterranean & $\mathrm{H}$ & A & M & $\mathrm{P}$ & $\mathrm{Ui}$ \\
\hline 69 & Cyperus difformis L. & Сyperaceae & Trop. America & $\mathrm{SE}$ & A & $\mathrm{Ch}$ & $\mathrm{CF}$ & $\mathrm{Ui}$ \\
\hline 70 & Cyperus iria L. & Cyperaceae & Trop. America & $\mathrm{SE}$ & A & Ch & $\mathrm{CF}$ & $\mathrm{Ui}$ \\
\hline 71 & Cytisus scoparius (L.) Link & Papilionaceae & Europe & $\mathrm{H}$ & A & M & $\mathrm{C}$ & $\mathrm{Ui}$ \\
\hline 72 & Datura fastuosa L. & Solanaceae & S. America, Mexico & $\mathrm{S}$ & $\mathrm{P}$ & $\mathrm{M}$ & $\mathrm{AR}, \mathrm{W}$ & $\mathrm{Ui}$ \\
\hline 73 & Datura innoxia Mill. & Solanaceae & Trop. America & $\mathrm{S}$ & $\mathrm{P}$ & $\mathrm{M}$ & $\mathrm{W}$ & $\mathrm{Ui}$ \\
\hline 74 & Datura metel L. & Solanaceae & Trop. America & $\mathrm{S}$ & $\mathrm{P}$ & $\mathrm{M}$ & $\mathrm{W}$ & $\mathrm{Ui}$ \\
\hline 75 & Datura stramonium L. & Solanaceae & Trop. America & $\mathrm{S}$ & $\mathrm{P}$ & $\mathrm{M}$ & $\mathrm{AR}, \mathrm{W}$ & $\mathrm{Ui}$ \\
\hline 76 & Dicoma tomentosa Cass. & Asteraceae & Trop. Africa & $\mathrm{H}$ & $\mathrm{A}$ & $\mathrm{Nk}$ & $\mathrm{W}$ & $\mathrm{Ui}$ \\
\hline
\end{tabular}




\section{Continued}

\begin{tabular}{|c|c|c|c|c|c|c|c|c|}
\hline 77 & Digera muricata (L.) Mart. & Amaranthaceae & S. W. Asia & $\mathrm{H}$ & A & $\mathrm{M}$ & $\mathrm{CF}$ & $\mathrm{Ui}$ \\
\hline 78 & Dinebra retroflexa (Valh) Panz. & Poaceae & Trop. America & G & $\mathrm{P}$ & Fo & $\mathrm{W}$ & $\mathrm{Ui}$ \\
\hline 79 & Echinochloa colona (L.) Link & Poaceae & S. America & G & A & Ft, Fo & $\mathrm{RB}$ & $\mathrm{Ui}$ \\
\hline 80 & Echinochloa crus-galli (L.) P. Beauv. & Poaceae & S. America & G & A & Ad & $\mathrm{RB}$ & $\mathrm{Ui}$ \\
\hline 81 & Echinops echinatus Roxb. & Asteraceae & Afghanistan & $\mathrm{H}$ & A & $\mathrm{Nk}$ & $\mathrm{W}$ & $\mathrm{Ui}$ \\
\hline 82 & Eclipta prostrata (L.) Mant. & Asteraceae & Trop. America & $\mathrm{H}$ & A & $\mathrm{Nk}$ & AR & $\mathrm{Ui}$ \\
\hline 83 & Eichhornia crassipes (C. Martius) Solms. & Pontederiaceae & Trop. America & $\mathrm{H}$ & $\mathrm{P}$ & Co, St & A & $\mathrm{O}$ \\
\hline 84 & Emilia sonchifolia (L.) DC. & Asteraceae & Trop. America & $\mathrm{H}$ & A & $\mathrm{M}, \mathrm{V}$ & $\mathrm{RB}$ & $\mathrm{Ui}$ \\
\hline 86 & Euphorbia chamaesyce L. & Euphorbiaceae & W. Africa and Mauritius & $\mathrm{H}$ & A & M & $\mathrm{W}$ & $\mathrm{Ui}$ \\
\hline 87 & Euphorbia cyathophora Murray & Euphorbiaceae & Trop. America & $\mathrm{H}$ & A & $\mathrm{Nk}$ & W & $\mathrm{Ui}$ \\
\hline 88 & Euphorbia heterophylla L. & Euphorbiaceae & Trop. America & $\mathrm{H}$ & A & $\mathrm{O}$ & CF & $\mathrm{Ui}$ \\
\hline 89 & Euphorbia hirta L. & Euphorbiaceae & Trop. America & $\mathrm{H}$ & A & $\mathrm{M}$ & $\mathrm{CF}$ & $\mathrm{Ui}$ \\
\hline 90 & Euphorbia peplus L. & Euphorbiaceae & Europe & $\mathrm{H}$ & $\mathrm{P}$ & M & $\mathrm{AR}, \mathrm{FE}$ & $\mathrm{Ui}$ \\
\hline 91 & Evolvulus nummularius (L.) L. & Convolvulaceae & Trop. America & $\mathrm{H}$ & $\mathrm{P}$ & $\mathrm{Ch}$ & $\mathrm{W}$ & $\mathrm{Ui}$ \\
\hline 92 & Fuirena ciliaris (L.) Roxb. & Cyperaceae & Trop. America & $\mathrm{SE}$ & A & $\mathrm{Nk}$ & W & $\mathrm{Ui}$ \\
\hline 94 & Galinsoga quadriradiata Ruiz \& Pavon & Asteraceae & Mexico & $\mathrm{H}$ & A & $\mathrm{Nk}$ & W & $\mathrm{Ui}$ \\
\hline 95 & Glossocardia bosvallea (L.f.) DC. & Asteraceae & East Indies & $\mathrm{H}$ & A & $\mathrm{Nk}$ & W & $\mathrm{Ui}$ \\
\hline 96 & Gnaphalium coarctatum Willd. & Asteraceae & Trop. America & $\mathrm{H}$ & A & $\mathrm{Nk}$ & $\mathrm{W}$ & $\mathrm{Ui}$ \\
\hline 97 & Gnaphalium pensylvanicum Willd. & Asteraceae & Trop. America & $\mathrm{H}$ & A & $\mathrm{Nk}$ & $\mathrm{RB}$ & $\mathrm{Ui}$ \\
\hline 98 & Gnaphalium polycaulon Pers. & Asteraceae & Trop. America & $\mathrm{H}$ & A & $\mathrm{Nk}$ & W & $\mathrm{Ui}$ \\
\hline 99 & Gomphrena celosioides Martius & Amaranthaceae & S. America & $\mathrm{H}$ & A & $\mathrm{Nk}$ & $\mathrm{CF}$ & $\mathrm{Ui}$ \\
\hline 100 & Gomphrena serrata L. & Amaranthaceae & Trop. America & $\mathrm{H}$ & A & $\mathrm{V}$ & $\mathrm{CF}$ & $\mathrm{Ui}$ \\
\hline 101 & Grangea maderaspatana (L.) Poir. & Asteraceae & S. America & $\mathrm{H}$ & A & $\mathrm{M}$ & $\mathrm{RB}$ & $\mathrm{Ui}$ \\
\hline 102 & Hyptis suaveolens (L.) Poit. & Lamiaceae & Trop. America & $\mathrm{H}$ & A & $\mathrm{M}$ & AR & $\mathrm{Ui}$ \\
\hline 103 & Impatiens balsamina $L$. & Balsaminaceae & Trop. America & $\mathrm{H}$ & A & $\mathrm{Cn}$ & $\mathrm{RB}$ & $\mathrm{O}$ \\
\hline 104 & Imperata cylindrical (L.) Raeusch. & Poaceae & Trop. America & G & $\mathrm{P}$ & $\mathrm{R}$ & W & $\mathrm{Ui}$ \\
\hline 105 & Indigofera astragalina DC. & Fabaceae & Trop. America & $\mathrm{H}$ & A & $\mathrm{Ch}$ & $\mathrm{F}$ & $\mathrm{Ui}$ \\
\hline 106 & Indigofera glandulosa Roxb. ex Willd. & Fabaceae & Trop. America & $\mathrm{H}$ & A & $\mathrm{M}$ & CF & $\mathrm{Ui}$ \\
\hline 107 & Indigofera linifolia (L.f.) Retz. & Fabaceae & S. America & $\mathrm{H}$ & A & M & AR & $\mathrm{Ui}$ \\
\hline 108 & Indigofera linnaei Ali & Fabaceae & Trop. Africa & $\mathrm{H}$ & A & M, Ma & $\mathrm{F}$ & $\mathrm{Ui}$ \\
\hline 109 & Indigofera trita L.f. & Fabaceae & Trop. Africa & $\mathrm{S}$ & $\mathrm{P}$ & $\mathrm{Ch}$ & $\mathrm{F}$ & $\mathrm{Ui}$ \\
\hline 111 & Ipomoea eriocarpa R.Br. & Convolvulaceae & Trop. Africa & $\mathrm{H}$ & A & M & $\mathrm{W}$ & $\mathrm{O}$ \\
\hline 112 & Ipomoea hederifolia L. & Convolvulaceae & Trop. America & $\mathrm{H}$ & A & $\mathrm{Ch}$ & $\mathrm{F}$ & $\mathrm{Ui}$ \\
\hline 113 & Ipomoea muricata (L.) Jacquin & Convolvulaceae & Trop. America & $\mathrm{H}$ & A & $\mathrm{M}$ & CF, W & $\mathrm{Ui}$ \\
\hline 114 & Ipomoea nil (L.) Roth & Convolvulaceae & N. America & $\mathrm{H}$ & A & $\mathrm{M}$ & FE, W & $\mathrm{Ui}$ \\
\hline 115 & Ipomoea obscura (L.) Ker.-Gawal. & Convolvulaceae & Trop. Africa & $\mathrm{H}$ & $\mathrm{P}$ & $\mathrm{M}$ & $\mathrm{W}$ & $\mathrm{Ui}$ \\
\hline 116 & Ipomoea pes-tigridis L. & Convolvulaceae & Trop. East Africa & $\mathrm{H}$ & A & $\mathrm{M}$ & $\mathrm{W}$ & $\mathrm{Ui}$ \\
\hline 117 & Ipomoea purpurea (L.) Roth & Convolvulaceae & S. America & $\mathrm{H}$ & A & M, Fo & $\mathrm{CF}, \mathrm{W}$ & $\mathrm{Ui}$ \\
\hline 118 & Ipomoea quamoclit L. & Convolvulaceae & Trop. America & $\mathrm{H}$ & $\mathrm{P}$ & M & W & $\mathrm{O}$ \\
\hline 119 & Ipomoea staphylina Roem. \& Schult. & Convolvulaceae & Trop. Africa & $\mathrm{H}$ & A & $\mathrm{Nk}$ & $\mathrm{W}$ & $\mathrm{Ui}$ \\
\hline 120 & Lagascea mollis Cav. & Asteraceae & Central America & $\mathrm{H}$ & A & $\mathrm{Nk}$ & $\mathrm{CF}$ & $\mathrm{Ui}$ \\
\hline 121 & Lantana camara L. & Verbenaceae & Trop. America & $\mathrm{H}$ & $\mathrm{P}$ & $\mathrm{Bf}, \mathrm{Sb}$ & $\mathrm{F}$ & $\mathrm{O}$ \\
\hline 122 & Leonotis nepetiifolia (L.)R. Br. & Lamiaceae & Trop. Africa & $\mathrm{H}$ & A & M & W & $\mathrm{Ui}$ \\
\hline 123 & Leucaena latisiliqua (L.) Gilli. & Mimosaceae & Trop. America & $\mathrm{H}$ & $\mathrm{P}$ & M & $\mathrm{W}$ & Fo \\
\hline 124 & Ludwigia adscendens (L.) Hara & Onagraceae & Trop. America & $\mathrm{H}$ & A & $\mathrm{Sb}$ & A & $\mathrm{Ui}$ \\
\hline 125 & Ludwigia octovalvis (Jacq.) Raven & Onagraceae & Trop. Africa & $\mathrm{H}$ & A & $\mathrm{M}$ & $\mathrm{RB}$ & $\mathrm{Ui}$ \\
\hline 126 & Ludwigia perennis L. & Onagraceae & Trop. Africa & $\mathrm{H}$ & A & M & $\mathrm{RB}$ & $\mathrm{Ui}$ \\
\hline 127 & $\begin{array}{l}\text { Macroptilium atropurpureum } \\
\text { (Mocino \& Sesse et DC) Urb. }\end{array}$ & Fabaceae & Trop. America & $\mathrm{H}$ & $\mathrm{P}$ & Fo & CF & $\mathrm{Ui}$ \\
\hline 128 & Macroptilium lathyroides (L.) Urb. & Fabaceae & C. \& S. America & $\mathrm{H}$ & A & $\mathrm{Nk}$ & W & $\mathrm{Ui}$ \\
\hline 129 & Malachra capitata (L.) L. & Malvaceae & Trop. America & $\mathrm{H}$ & $\mathrm{P}$ & M & W & $\mathrm{Ui}$ \\
\hline 130 & Malvastrum coromandelianum (L.) Garcke & Malvaceae & Trop. America & $\mathrm{H}$ & A & $\mathrm{M}, \mathrm{Fi}$ & W & $\mathrm{Ui}$ \\
\hline 131 & Martynia annua L. & Pedaliaceae & Trop. America & $\mathrm{H}$ & $\mathrm{P}$ & M & W & $\mathrm{Ui}$ \\
\hline 132 & Mecardonia procumbens (Mill.) Small & Scrophulariaceae & Trop. North America & $\mathrm{H}$ & A & $\mathrm{Ch}$ & $\mathrm{W}$ & $\mathrm{Ui}$ \\
\hline 133 & Melilotus alba Medik. ex Desr. & Fabaceae & Europe & $\mathrm{H}$ & A & $\mathrm{Fo}, \mathrm{Br}$ & $\mathrm{CF}$ & $\mathrm{Fd}$ \\
\hline 134 & Melochia corchorifolia L. & Sterculiaceae & Trop. America & $\mathrm{H}$ & $\mathrm{P}$ & $\mathrm{Fi}, \mathrm{V}$ & $\mathrm{F}$ & $\mathrm{Ui}$ \\
\hline 135 & Merremia aegyptia (L.) Urb. & Convolvulaceae & Trop. America & $\mathrm{C}$ & $\mathrm{P}$ & $\mathrm{Nk}$ & W & $\mathrm{Ui}$ \\
\hline 136 & Mimosa pudica L. & Mimosaceae & Brazil & $\mathrm{H}$ & $\mathrm{P}$ & M & $\mathrm{F}$ & $\mathrm{Ui}$ \\
\hline 137 & Mirabilis jalapa L. & Nyctaginaceae & Peru & $\mathrm{H}$ & A & $\mathrm{O}$ & $\mathrm{W}$ & $\mathrm{O}$ \\
\hline 138 & Monochoria vaginalis (Burm.f.) C. Presl. & Pontederiaceae & Trop. America & $\mathrm{H}$ & $\mathrm{P}$ & $\mathrm{M}, \mathrm{V}$ & $\mathrm{RB}$ & $\mathrm{Ui}$ \\
\hline 139 & Nicotiana plumbaginifolia Viv. & Solanaceae & Trop. America & $\mathrm{H}$ & A & $\mathrm{Sm}$ & $\mathrm{W}$ & $\mathrm{Ui}$ \\
\hline
\end{tabular}




\section{Continued}

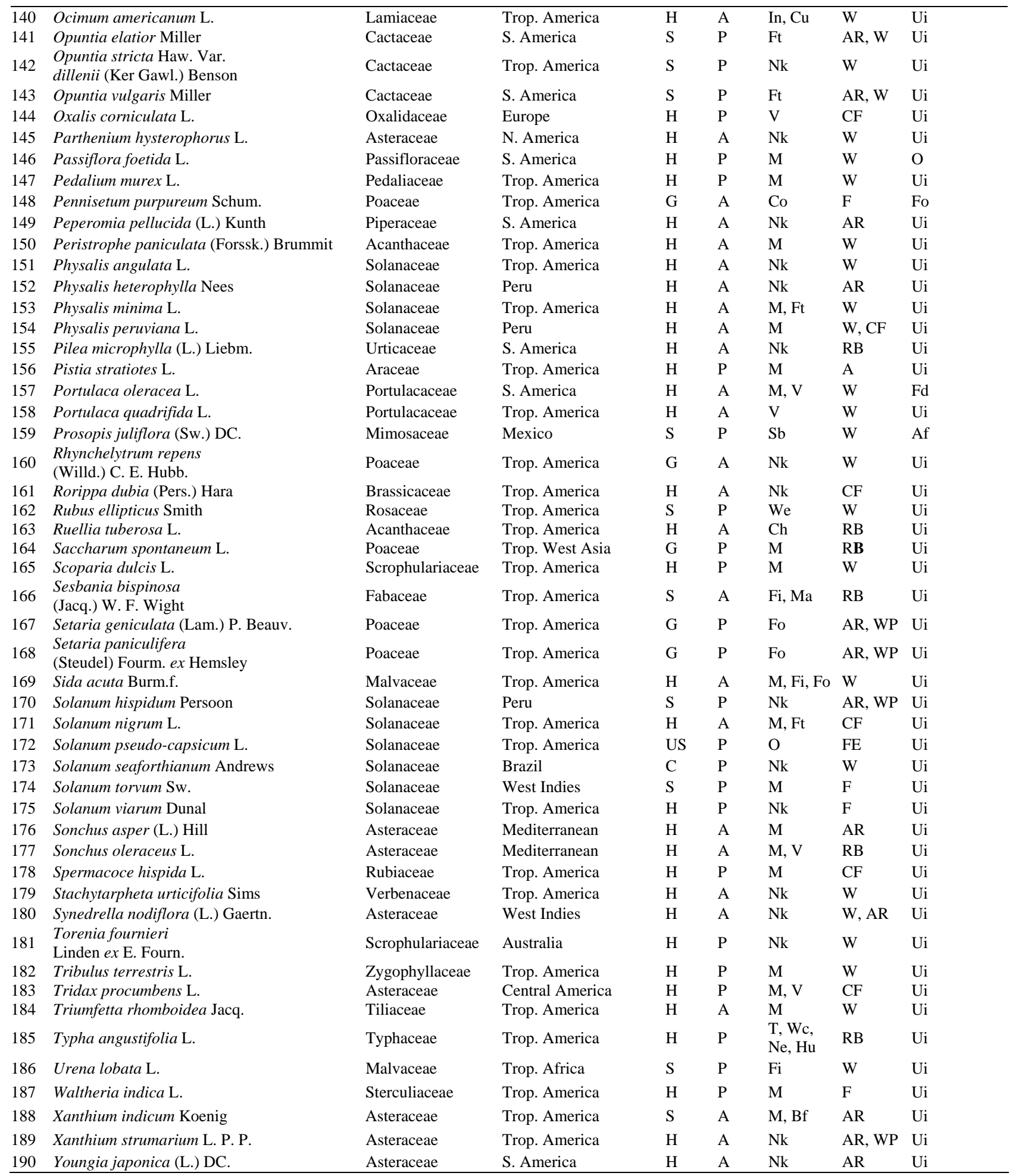

Life form: H-Herb; C_Climber; US-Undershrub; S—Shrub; SE—Sedges; T-Tree; G_Grass.

Habit: A-Annual; P-Perennial.

Uses: Ad-Adulteration; B-Basket making; Bf-Biomass fuel in rural area; $\mathrm{Br}$-Biofertilizer; C—Clothes making; Ch-Presence of bioactive chemicals; Cn-Condiment; Co-Compost; Cu_Culinery; Fi-Fibre; Fo-Fodder; Ft—Fruits edible; Hu—Hut; Hf—Hand-held fan; In-Insectiside; M-Medicinal; Ma—Manure; Ne—Net making; Nk—Not known; O—Ornamental; P—Poisonous plant; R—Rope making; Sa—Sacred Plant; Sb—Soil binder; Sc—Sac making; Sm—Smoking; So—Social forestry, St—Secondary waste water treatment; T—Thatching; V—Vegetable; W-Wood work; Wc-Wicker work; We-Wild edible. Habitat: W-Wastelands; CF-Cultivated fields; F-Forests; AR-Along roadside; A-Aquatic; P—Parasites; CF—Crop fields; RB-River beds.

Mode of introduction: Af_-Agroforestry; Fd—Food; Fo—-Fodder; O_Ornamental; Ui_-Unintentional. 


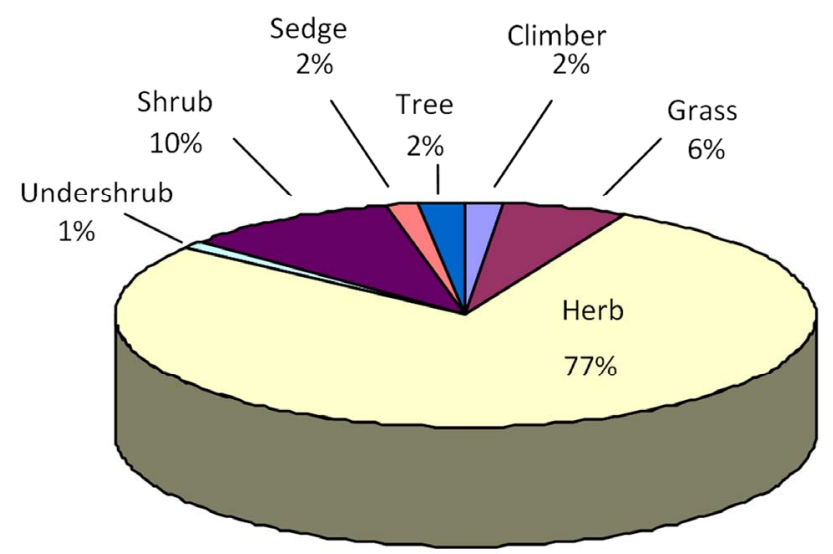

Figure 1. Life forms of invasive species.

Euphorbia, Indigofera and Datura (4 sp. each). These top 9 genera contributed 26\% taxa of alien flora of IHR. The family Asteraceae is the most dominant family with 31 species; followed by Solanaceae (15 sp.); Convolvulaceae and Fabaceae (13 sp. each); Amaranthaceae and Poaceae (11 sp. each); Caesalpiniaceae (9 sp.); Euphorbiaceae (7 sp.); Mimosaceae and Tiliaceae (6 sp. each). These ten dominant families contributed $64 \%$ of the alien invasive flora of IHR (Figure 2).

About $54 \%$ of invasive species were most abundant in wastelands, while cultivated fields, road sides, river beds, forest/forest edges were favored by $17 \%, 15 \%, 10 \%$ and $9 \%$ respectively. A search of literature, herbarium and consultation with local people indicated that several of the invasive species are also being used for different purposes for example, the stem of Malvastrum coromandelianum, Sesbania bispinosa, Sida acuta, and Cannabis sativa is being used for fibre, and Borassus flabellifer is being used for Hand-held fan. Ninety species listed in Table 1 are reported to be used by locals for medicinal purposes. The species namely, Leucaena leucocephala is being effectively used for social forestry. The uses of twenty seven species are not known or even not used by locals. Five plants are reported having high allelopathic potential, namely, Echinochloa crusgalli, Lagascea mollis, Lantana camara, Parthenium hysterophorus and Prosopis juliflora.

\section{Discussion}

Alien species are non-native or exotic organisms that occur outside their natural adapted ranges and dispersal potential [6]. Many alien species support our farming and forestry systems in a big way. However, some of the alien species become invasive when they are introduced deliberately or unintentionally outside their natural habitats into new areas where they express the capability to establish, invade and out-compete native species [3]. International Union for Conservation of Nature and

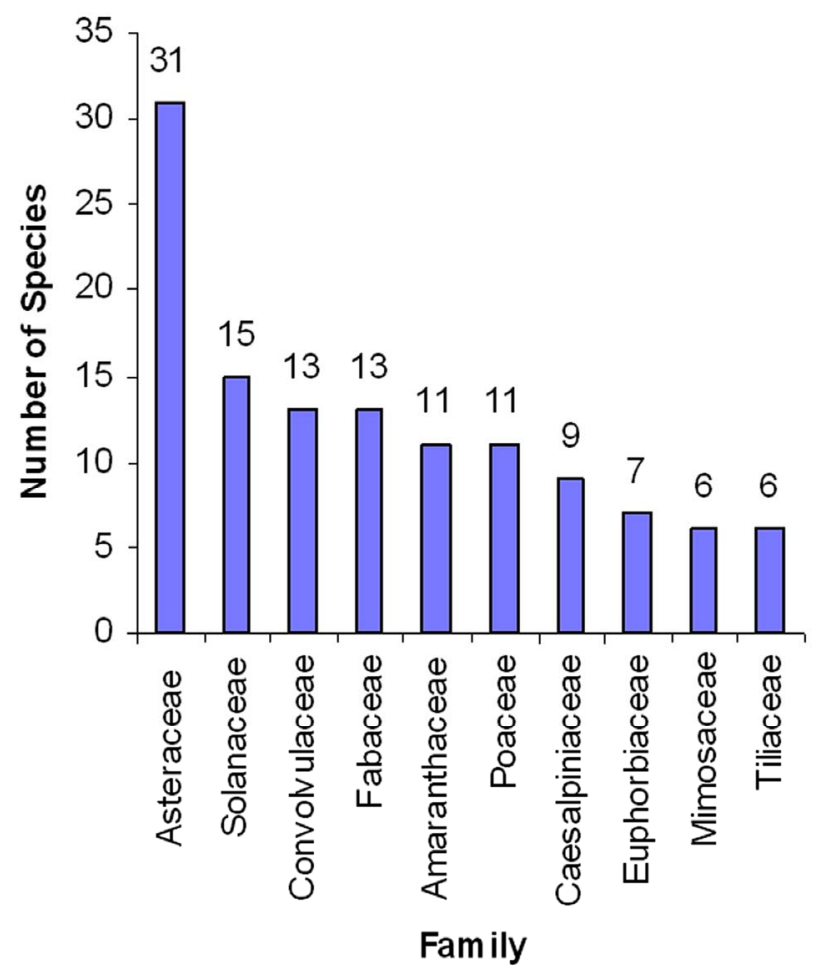

Figure 2. Ten dominant families of invasive species.

Natural Resources (IUCN) defines Alien Invasive Species as an alien species which becomes established in natural or semi-natural ecosystems or habitat, an agent of change, and threatens native biological diversity. These invasive are widely distributed in all kinds of ecosystems throughout the world, and include all categories of living organisms. Nevertheless, plants, mammals and insects comprise the most common types of invasive alien species in terrestrial environments [3]. In Indian Himalayan Region, comprehensive studies on invasive species and plant invasions are still missing. In view of this, the present study attempted to focus on document the invasive alien species in the flora of IHR. This listed invasive exotic species will serve as basic information for future research towards the conservation of endemic and natural forest vegetation of Himalaya.

In IHR, 190 species distributed in 112 genera and 47 families recorded as invasive alien in the flora. Amongst the recorded invasive species, many plants are known invasive of nearby region also. For example, 124 species listed in table 1 are common invasive in the state of Uttar Pradesh [35]. Among these invasive species of IHR, 73\% are native to American continent. The invasive flora of India also concerns more than $58 \%$ of invasive plants are native to American continent [34,35]. The lesser percentage of American elements in IHR may be due to the presence of high altitudes, as the Europian flora contributed maximum percentage of invasive species in Kashmir Himalaya [37]. Annuals are recorded the predomi- 
nant invasive flora of IHR (63\%), so the habit-wise classification of alien invasive shows a preponderance of herbs (77\%). Further, greater viability and tolerance to harsh conditions could result this preponderance of herbs in the alien flora. Asteraceae is the dominant invasive family of IHR and contributed most of the exotic weed species in our country [34]. Singh et al. [35] also recorded the dominance of Asteraceae in invasive alien flora of Uttar Pradesh and Rao and Murugan [37] also found that the Asteraceae is dominating family in alien flora of India. It is obvious that the family Fabaceae is one among the largest families of alien flora of IHR and having the potential of Nitrogen fixing capacity would be helpful to them in colonizing the empty niches. It is obviously recorded that 47 percent of the invasive species are under the consideration of medicinal purposes. But some of the species like Echinochloa crus-galli, Lagascea mollis, Lantana camara and Parthenium hysterophorus are having high allelopathic potential and harmful to natural plant population [35].

The invasive species cause loss of biodiversity including species extinctions, and changes in hydrology and ecosystem function. Differences between native and exotic plant species in their requirements and modes of resource acquisition and consumption may cause a change in soil structure, its profile, decomposition, nutrient content of soil, moisture availability, etc. Invasive species are thus a serious hindrance to conservation and sustainable use of biodiversity, with significant undesirable impacts on the goods and services provided by ecosystems. Biological invasions now operate on a global scale and will undergo rapid increase in this century due to interaction with other changes such as increasing globalization of markets, rise in global trade, travel and tourism. For effective management of invasive species, knowledge about their ecology, morphology, phenology, reproducetive biology, physiology and phytochemistry is essential [3]. Monitoring of invasion can be done through qualitative approach like species inventory (seasonally) and quantitative approach using phytosociological methods and mapping using ground-based methods (via map overlays or GPS), remotely-sensed images (aerial photos, high resolution multi-spectral digital data). A better planning is needed for early detection and reporting of infestations of spread of new and naturalized weeds by creation of plant detection network in IHR by establishing communication links between taxonomists, ecologists and land managers to monitor and control.

\section{Acknowledgements}

The authors thankful to Dr. L. M. S. Palni, Director, G. B. Pant Institute of Himalayan Environment \& Development, Kosi-Katarmal, Almora for providing facilities and encouragements. Sincere thanks to Dr. R. S. Rawal, Sci-
entist—E, BCM Theme, G. B. Pant Institute of Himalayan Environment \& Development, Kosi-Katarmal, Almora for constant support.

\section{REFERENCES}

[1] Anonymous, Indian Himalayan Region, ENVIS Centre on Himalayan Ecology, G. B. Pant Institute of Himalayan Environment \& Development, Almora, 2011.

[2] S. S. Samant, U. Dhar and L. M. S. Palni, "Medicinal Plants of Indian Himalaya: Diversity, Distribution and Potential Value,” G. B. Pant Institute of Himalayan Environment \& Development, Almora, 1998.

[3] A. S. Raghubanshi, L. C. Rai, J. P. Gaur and J. S. Singh, "Invasive Alien Species and Biodiversity in India," Current Science, Vol. 88, No. 4, 2005, pp. 539-540.

[4] Y. H. Sujay, H. N. Sattagi and R. K. Patil, "Invasive Alien Insects and Their Impact on Agroecosystem," Karnataka Journal of Agricultural Sciences, Vol. 23, No. 1, 2010, pp. 26-34.

[5] H. M. Pant and N. Sharma, "Inventory of Some Exotic Cultivated Tree Species of Doon Valley and Their Ethnobotanical Uses,” Journal of Medicinal Plants Research, Vol. 4, No. 20, 2010, pp. 2144-2147.

[6] M. A. McGeoch, S. H. M. Butchart, D. Spear, E. Marais, E. J. Kleynhans, A. Symes, J. Chanson and M. Hoffmann, "Global Indicators of Biological Invasion: Species Numbers, Biodiversity Impact and Policy Responses,” Diversity and Distributions, Vol. 16, No. 1, 2010, pp. 95-108. doi:10.1111/j.1472-4642.2009.00633.x

[7] M. E. Eiswerth, T. D. Darden, W. S. Johnson, J. Agapoff and R. H. Thomas, "Input-Output Modeling, Outdoor Recreation, and the Economic Impacts of Weeds," Weed Science, Vol. 53, No. 1, 2005, pp. 130-137. doi:10.1614/WS-04-022R

[8] H. Hara, Ed., "Flora of Eastern Himalaya, Second Report,” University of Tokyo Press, Tokyo, 1971.

[9] C. R. Babu, "Herbaceous Flora of Dehradun," Council of Scientific and Industrial Research, New Delhi, 1977.

[10] H. J. Chowdhery and B. M. Wadhwa, "Flora of Himachal Pradesh-Analysis,” Vol. 1-3, Botanical Survey of India, Howrah, 1984.

[11] R. K. Gupta, “The Living Himalaya,” Vol. I, Today and Tomorrow Publication, New Delhi, 1983.

[12] R. K. Gupta, “The Living Himalaya,” Vol. II, Today and Tomorrow Publication, New Delhi, 1989.

[13] H. J. Chowdhery, G. S. Giri, G. D. Pal, A. Pramanik and S. K. Das, "Materials for the Flora of Arunachal Pradesh," Vol. I, Botanical Survey of India, Calcutta, 1996, pp. 1693.

[14] H. J. Chowdhery, G. S. Giri, G. D. Pal, A. Pramanik and S. K. Das, "Materials for the Flora of Arunachal Pradesh,” Vol. 2, Botanical Survey of India, Calcutta, 2008, pp. 1-670.

[15] R. C. Srivastava, "Flora of Sikkim (Ranunculaceae-Moringaceae),” Oriental Enterprises, Delhi, 1998.

[16] R. D. Gaur, "Flora of the District Garhwal, North West 
Himalaya (with Ethnobotanical Notes)," TransMedia, Srinagar, Garhwal, 1999.

[17] S. Kumar and V. Singh, “Asteraceae of Sikkim,” Deep Publications, New Delhi, 2001.

[18] N. P. Singh, D. K. Singh and B. P. Uniyal, Eds., "Flora of Jammu \& Kashmir,” Vol. 1, Botanical Survey of India, Kolkata, 2002.

[19] P. K. Hajra and A. De, “The Indigenous and Exotic Beauties of Dehradun,” Oriental Enterprises, Dehradun, 2007.

[20] J. D. Hooker, "Flora of British India,” Vol. 1-7, Reeve \& Co. Ltd., London, 1872-1897.

[21] B. P. Uniyal, J. R. Sharma, U. Choudhery and D. K. Singh, "Flowering Plants of Uttarakhand," Bishen Singh Mahendra Pal Singh, Dehradun, 2007.

[22] J. K. Maheshwari, "Studies on the Naturalized Flora of India," Proceedings of the Summer School of Botany, New Delhi, 2-15 June 1960, pp. 156-170.

[23] K. M. Matthew, "Alien flora of Kodai Kanal and Palni Hills," Records of Botanical Survey of India, Vol. 20, No. 1, 1969, pp. 1-241.

[24] J. K. Maheswari and S. R. Paul, "The Alien Flora of Ranchi,” Journal of the Bombay Natural History Society, Vol. 72, No. 1, 1975, pp. 158-188.

[25] M. P. Nayar, "Changing Patterns of the Indian Flora," Bulletin of Botanical Survey of India, Vol. 19, No. 1-4, 1977, pp. 145-155.

[26] P. K. Hajra and B. K. Das, "Vegetation of Gangtok with Special Reference to Alien Plants," India Forums, Vol. 107, 1982, pp. 554-566.

[27] B. D. Sharma, "Exotic Flora of Allahabad," Botanical Survey of India, Dehra Dun, 1984.

[28] K. G. Saxena, "Biological Invasion in the Indian SubContinent: Review of Invasion by Plants,” In: P. S. Ramakrishnan, Ed., Ecology of Biological Invasion in the
Tropics, International Scientific Publications, New Delhi, 1991, pp. 53-73.

[29] R. P. Pandey and P. J. Parmar, "The Exotic Flora of Rajasthan," Journal of Economic and Taxonomic Botany, Vol. 18, No. 1, 1994, pp. 105-121.

[30] H. A. Mooney and R. J. Hobbs, "Invasive Species in a Changing World,” Island Press, Washington, 2000.

[31] D. S. Pandey, "Exotics-Introduced and Natural Immigrants, Weeds, Cultivated, etc.,” In: N. P. Singh, D. K. Singh, P. K. Hajra and B. D. Sharma, Eds., Flora of India: Introductory Volume, Part II, Botanical Survey of India, Calcutta, 2000, pp. 266-301.

[32] J. A. McNeely, H. A. Mooney, L. E. Neville, P. Schei and J. K. Waage, "A Global Strategy on Invasive Alien Species," IUCN Gland, Switzerland, and Cambridge, in Collaboration with the Global Invasive Species Programme, 2001.

[33] P. S. Negi and P. K. Hajra, “Alien Flora of Doon Valley, North West Himalaya,” Current Science, Vol. 92, No. 7, 2007, pp. 968-978.

[34] C. S. Reddy, "Catalogue of Invasive Alien Flora of India,” Life Science Journal, Vol. 5, No. 2, 2008, pp. 84-89.

[35] K. P. Singh, A. N. Shukla and J. S. Singh, "State-Level Inventory of Invasive Alien Plants, Their Source Regions and Use Potential,” Current Science, Vol. 99, No. 1, 2010, pp. 107-114.

[36] A. A. Khuroo, I. Rashid, Z. Reshi, G. H. Dar and B. A. Wafai, “The Alien Flora of Kashmir Himalaya,” Biological Invasions, Vol. 9, No. 3, 2007, pp. 269-292. doi:10.1007/s10530-006-9032-6

[37] R. R. Rao and R. Murugan, "Impact of Exotic Adventives Weeds on Native Biodiversity in India: Implications for Conservation,” In: L. C. Rai and J. P. Gaur, Eds., Invasive Alien Species and Biodiversity in India, Banaras Hindu University, Varanasi, 2006, pp. 93-109. 